\title{
THEORETICAL MODELING OF DEFECTS IN THE MOLECULAR CRYSTAL OF 2-(2'-HYDROXYPHENYL)BENZOTHIAZOLE
}

\author{
Y. Syetov \\ Oles Honchar Dnipro National University, Dnipro, Ukraine \\ e-mail: setov2003@yahoo.com
}

\begin{abstract}
2-(2'-hydroxyphenyl)benzothiazole is a photoreactive compound that exhibits excited state intramolecular proton transfer in the structure with an $\mathrm{OH} . . \mathrm{N}$ hydrogen bond. Energy of various structures is calculated for isolated molecules, clusters and periodic structures of 2-(2'hydroxyphenyl)benzothiazole by density-functional based tight-binding methods. It is shown that the most stable conformation of the isolated molecule is a planar structure with an $\mathrm{OH}$...N hydrogen bond. Other conformations have significantly larger energy in comparison with the average room temperature heat energy that implies a low equilibrium number of those structures in non-polar solvents. In crystal the defect with lowest energy is non-hydrogen-bonded conformation formed by rotation of the $\mathrm{OH}$ bond. The energy of this defect is close to the energy difference for corresponding conformations of the isolated molecule. For other conformations, the energy values of the defects are larger than the energy differences for isolated molecules. In contrast to the crystal of 2-(2'-hydroxyphenyl)benzoxazole, energy of the defect caused by the entire molecule reorientation is comparable with the energy of defects caused by different conformations. method.

Keywords: molecular crystal, excited state proton transfer, defects, density-functional based tight-binding
\end{abstract}

Received 11.06.2020; Received in revised form 12.08.2020; Accepted 04.09.2020

\section{Introduction}

Photoinduced excited state intramolecular proton transfer (ESIPT) is studied for the last decades as an ultrafast photochemical reaction and a process that could be utilized for several applications in optoelectronics [1]. Since early 1970s 2-(2'-hydroxyphenyl)benzothiazole (HBT, Fig. 1) [2] is extensively studied as a model compound undergoing ESIPT. HBT is also considered as a substance which is suitable for materials in optoelectronics, one of the possible applications is laser generation by optically pumped nanowires [3]. In contrast to its benzoxazole analog, i.e. 2-(2'-hydroxyphenyl)benzoxazole (HBO) [4], a crystal of HBT does not demonstrate disorder in X-ray measurements [5, 6]. According to the X-ray data [6], HBT crystal belongs to the monoclinic P $2_{1} / \mathrm{c}$ structure class with 4 molecules in a unit cell (Fig. 2). Theoretical calculations show that the most stable structure of HBT molecule in the ground state is enol with an intramolecular $\mathrm{OH}$... N hydrogen bond (structure I in Fig. 1), whereas the structure IV is non-planar and unstable [7]. Electronic density in the molecule of HBT undergoes redistribution upon absorbing photon so that a keto structure becomes energetically favorable in the excited state. Transition to the ground state in the keto structure causes fluorescence with an anomalously large Stokes shift. In an isolated molecule of HBT the ESIPT is followed by internal rotation in the keto structure that leads to radiationless transition to the ground state [8]. It is supposed that crystal packing restricts the intramolecular rotation and formation of the trans-enol conformation; however, amplified emission is obtained at two wavelengths and attributed to the formation of planar and partially twisted keto structures [3]. Modeling of point defects in the crystal lattice of HBO demonstrates that there are possible defects caused both by different orientation of the molecule with an $\mathrm{OH} \ldots \mathrm{N}$ hydrogen bond and by formation of structure with an $\mathrm{OH} \ldots \mathrm{O}$ hydrogen bond. The orientational defects are found to have lower energy than the $\mathrm{OH}$... rotamer [9].

In the present paper we describe results of theoretical modeling of crystal lattice of HBT aimed to clarify influence of crystal packing on structure of the molecules. 


\section{Calculations details}

Geometrical parameters of model structures of an HBT crystal were calculated by density functional based tight-binding (DFTB) method with the third order correction and van der Waals interaction correction implemented in the DFTB+ program package [10, 11] as described in [12]. Supercell folding is used for the Brillouin zone sampling for periodic structures, $3 \times 3 \times 3$ supercell is considered for the unit cell of the crystal lattice and $2 \times 2 \times 2$ - for the model structure. The structure is optimized for the Brillouin zone center assuming the cell parameters be fixed to the values taken from the X-ray data [6]. In the case of $\mathrm{HBO}$, this calculation method demonstrates reasonable agreement between calculated frequencies of low-frequency lattice vibrations and positions of Raman bands in the experimental spectrum [12]. The DFT calculations for isolated molecules were also performed with the GAMESS program suite $[13,14]$ using the B3LYP functional and 6$31 \mathrm{G}(\mathrm{d}, \mathrm{p})$ basis set. The energy of defects caused by reorientation of the molecule in the lattice or different conformation of the molecule is evaluated by calculations for the model clusters extracted from the optimized structure of a fully ordered crystal. The cluster includes the molecule under consideration and the nearest neighbors (Fig. 3). The positions of the atoms constituting the central molecule are optimized regarding the energy minimum. The coordinates of the atoms in the neighboring molecules are kept constant.

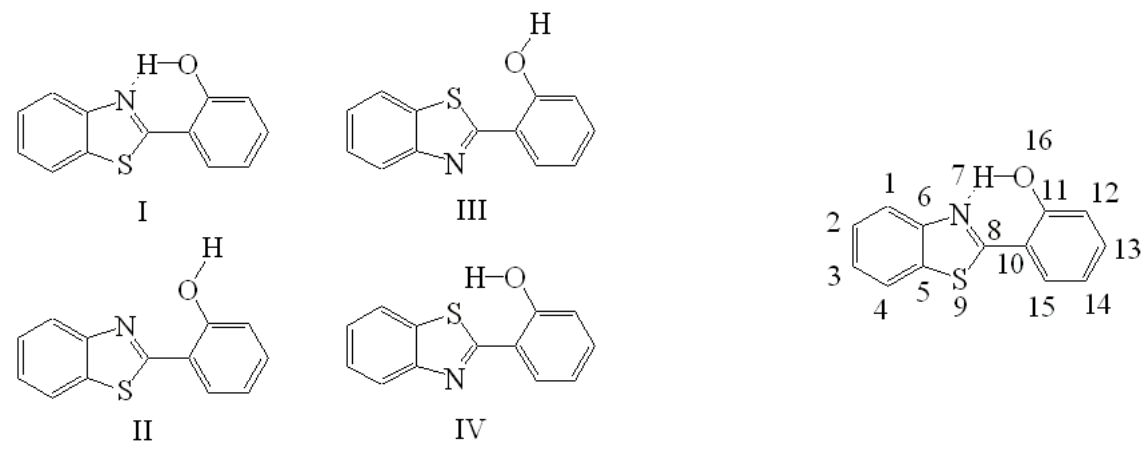

Fig. 1. Enol structures (I-IV) and atomic numbering scheme of molecules of HBT.
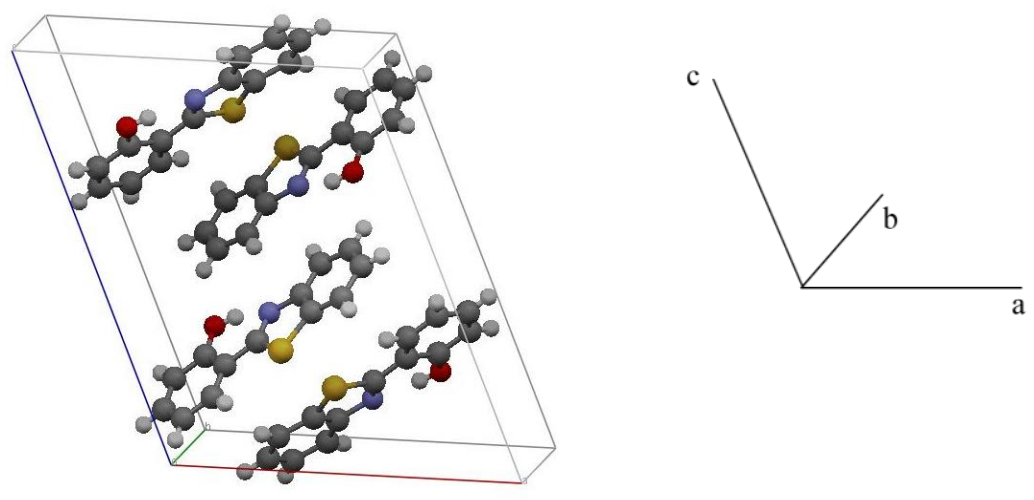

Fig. 2. Unit cell of a crystal of HBT. The unit cell is plotted according to the X-ray data [6]. 


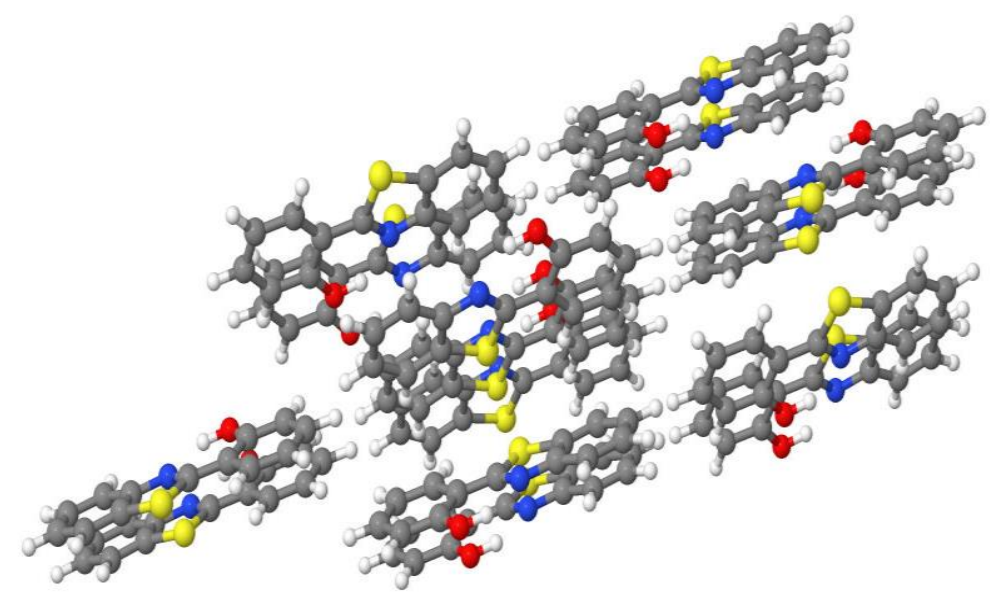

Fig. 3. Molecular cluster for modeling of defects in the crystal of HBT.

The periodic model structure of the defect comprises two unit cells of the crystal obtained by a translation along the lattice vector b (Fig. 4). One molecule in this structure is supposed to be oriented improperly or has different conformation. Translation vectors of the periodic structure are $\mathbf{a}, 2 \mathbf{b}$, and $\mathbf{c}$. The structure is chosen to provide the minimal number of molecules within the first coordination sphere for the defect molecule.
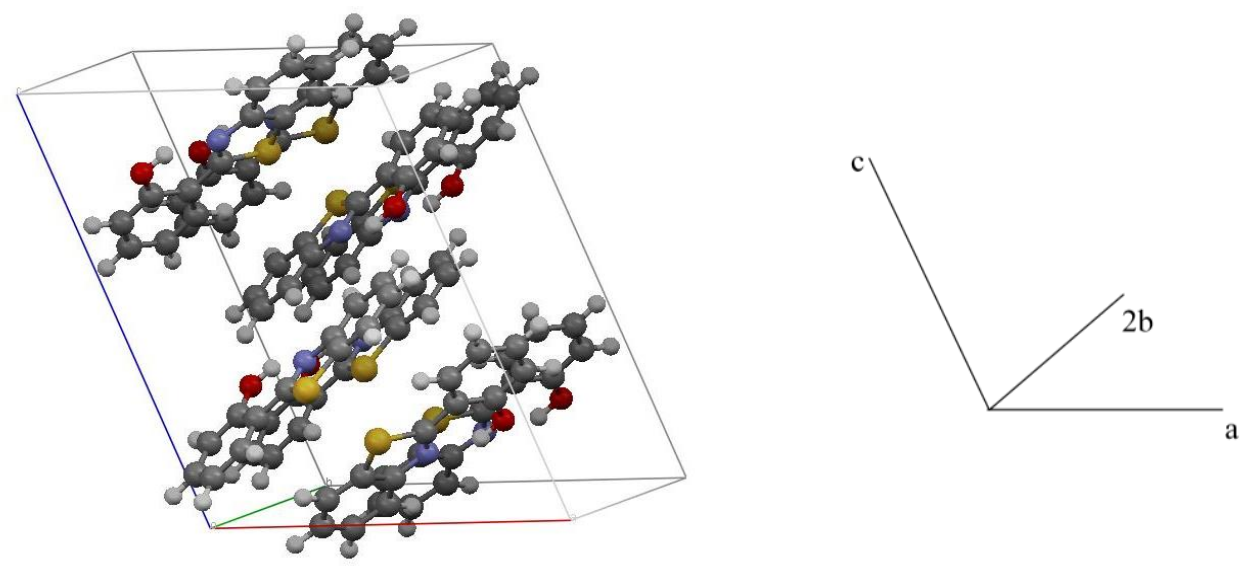

Fig. 4. Unit cell of the periodic structure for modeling of defects in the crystal of HBT.

\section{Results and discussion}

Values of energy of various structures corresponding to the energy minima are collected in Table 1. The calculations show that the most stable enol structure of an isolated molecule of HBT is the planar structure I with an intramolecular hydrogen bond $\mathrm{OH} . . . \mathrm{N}$. Structure IV is found to be non-planar with the dihedral angle between the benzothiazole and phenol moieties about $154^{\circ}$ (DFT) and $151^{\circ}$ (DFTB) (Fig. 5a). DFT calculations show that energy of structure IV is significantly larger than energy of the most stable structure I (see Table 1) and larger by about $0.03 \mathrm{eV}$ than energy of structure 
III. Structure III is an "open" conformation of the molecule that does not possess an intramolecular hydrogen bond. Another "open" structure II is predicted to be non-planar (Fig. 5b) by DFT calculations with even larger energy. The planar structure II obtained by the restriction to $C_{s}$ symmetry differs only by $0.02 \mathrm{eV}$ in energy from the non-planar structure that corresponds to the energy minimum. DFTB calculations yield the planar structure II, underestimate energy differences and predict larger energy for structure IV than for structure II (see Table 1). Nevertheless, both DFT and DFTB calculations demonstrate that structure with the lowest energy (except structure I) is structure III without the hydrogen bond rather than structure IV. DFT value of about $0.4 \mathrm{eV}$ for energy of structure III implies low equilibrium number (of the order of $10^{-7}$ ) of this species at room temperature.

Table 1

The calculated energy of various structures comprising molecules of HBT (in relation to the minimal energy)

\begin{tabular}{|c|c|c|c|c|c|c|c|c|}
\hline & \multicolumn{4}{|c|}{ Molecule } & \multicolumn{2}{c|}{ Cluster } & \multicolumn{2}{c|}{ Periodic srtucture } \\
\hline Structure & DFT & $\begin{array}{c}\mathrm{C}_{\mathrm{s}} \\
\text { restricted }\end{array}$ & DFTB & $\begin{array}{c}\mathrm{C}_{\mathrm{s}} \\
\text { restricted }\end{array}$ & & $\begin{array}{c}\text { Different } \\
\text { orientation }\end{array}$ & & $\begin{array}{c}\text { Different } \\
\text { orientation }\end{array}$ \\
\hline I & 0 & - & 0 & - & 0 & 0.50 & 0 & 0.38 \\
\hline II & 0.56 & 0.58 & 0.21 & - & 0.22 & - & 0.21 & - \\
\hline III & 0.39 & - & 0.18 & - & 0.35 & - & - & - \\
\hline IV & 0.42 & 0.52 & 0.25 & 0.26 & 0.47 & 0.69 & 0.43 & - \\
\hline
\end{tabular}

The DFTB calculations show that molecular units in the crystal lattice are close to planarity, the $\mathrm{N}_{7}-\mathrm{C}_{8}-\mathrm{C}_{10}$ (see Fig. 1) dihedral angle is about $6^{\circ}$. The corresponding experimental value from $\mathrm{X}$-ray data [6] is about $2^{\circ}$. Reorientation of the molecule that has structure I significantly increases energy of the cluster and periodic structure (see Table 1). Consideration of dimers extracted from the model cluster reveals that the largest contribution to the increase originates from the dimer formed by molecules along crystal axis b (Fig. 6).

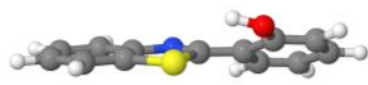

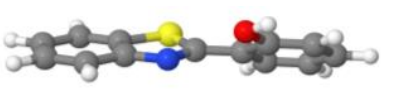

b

Fig. 5. Twisted structures IV (a) and II (b) obtained by the DFT calculations for molecules of HBT.

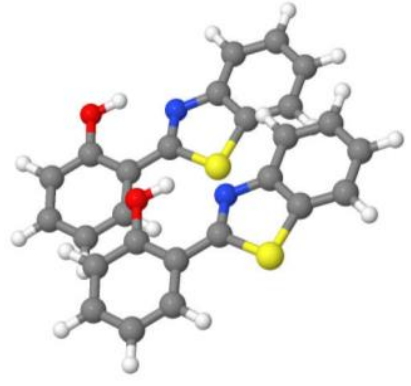

a

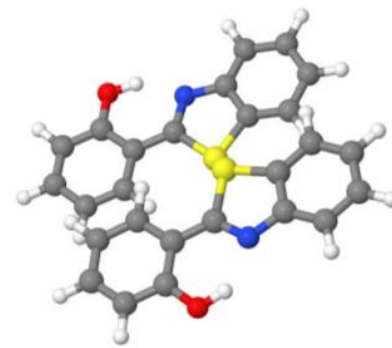

b

Fig. 6. The dimer in the model clusters demonstrating the largest change in energy: ordered structure (a) and different orientation of the molecule (b). 
Interaction of structure IV with the crystal environment leads to the planar conformation instead of the twisted structure of the isolated molecule. The energy difference for cluster and periodic structure increases in comparison with the energy difference between structures IV and I of the isolated molecule even for the planar structure IV obtained by restricted optimization. If structure IV in the crystal corresponds to different orientation of the benzothiazole moiety, then the energy difference is larger (see Table 1). The DFTB calculations on the model clusters show that "open" structure II only slightly (by about $0.01 \mathrm{eV}$ ) changes energy in comparison with the isolated molecule while structure III increases energy by about $0.17 \mathrm{eV}$. The close energy difference is also yielded by the calculations on the periodic structure and isolated molecule with structure II. It should be noted that structures II-IV do not undergo the excited state intramolecular proton transfer.

Thus, the calculations show that the defect energy caused by different orientation of the molecule is comparable with energy of the defects caused by its different conformations. The lowest energy defect is predicted to be structure II without an intramolecular hydrogen bond. The energy of this defect estimated by the DFTB calculations implies low equilibrium concentration $\left(10^{-4}\right)$ that is in good correspondence to the ordered crystal structure of HBT found by the X-ray diffraction measurements $[5,6]$.

\section{Conclusions}

Calculations with density-functional based tight-binding methods are performed for model clusters and periodic structures corresponding to the arrangement of molecules in the crystal lattice of 2-(2'-hydroxyphenyl)benzothiazole. The calculations demonstrate that energy of defects caused by a different orientation of a molecule or its different conformation is sufficient for low equilibrium concentration at room temperature. The calculations do not find out twisted enol structure of molecules in the ideal crystal lattice, the molecules are close to planarity in the ground state. The main drawback of the model is a small size of region surrounding the defect so that the resulting periodic structure is assumed to have the regular arrangement of the defect molecule in each two unit cell of the crystal. The considered structures comprise the minimal number of molecules providing that the defect molecule is surrounded by dominating species. Evaluation of the possible distortion of luminescent keto structures requires description of the intermolecular potential in the excited state. In the used model the intermolecular forces are described by an empirical atom-atom potential, which is parameterized only for the ground state.

\section{References}

1. Hsieh, C.-C. Organic Dyes with Excited-State Transformations (Electron, Charge, and Proton Transfers) / C.-C. Hsieh, M.-L. Ho, P.-T. Chou // Advanced Fluorescence Reporters in Chemistry and Biology I: Fundamentals and Molecular Design / Ed. by A.P. Demchenko. - Springer Ser. Fluoresc. - 2010. - Vol. 8. - P. 225 266.

2. Williams, D.L. Intramolecular proton transfer reactions in excited fluorescent compounds / D.L. Williams, A. Heller // J. Phys. Chem. - Vol. 74. - 1970. - P. 4473 4480 .

3. Zhang, W. Low-Threshold Wavelength-Switchable Organic Nanowire Lasers Based on Excited-State Intramolecular Proton Transfer / W. Zhang, Y. Yan, J. Gu, J. Yao, Y. S. Zhao // Angew. Chem. Int. Ed. - 2015. - Vol. 54. - P. 7125 - 7129. 
4. Tong, Y.-P. 2-(2-Hydroxyphenyl)-1,3-benzoxazole / Y.-P. Tong // Acta Cryst. E. - 2005. - Vol. 61. - P. 03076 - 03078.

5. Sténson, P. The Crystal Structure of 2-(o-Hydroxyphenyl)benzothiazole / P. Sténson // Acta Chem. Scand. - 1970. - Vol. 24. - P. 3729 - 3738.

6. Jia, A.-Q. Syntheses, characterization, and ethylene polymerization of titanium and zirconium complexes with [N, O] ligands / A.-Q. Jia and G.-X. Jin // Dalton Trans. 2009. - P. 8838 - 8845. CCDC 727487: Experimental Crystal Structure Determination. 2010. - DOI: $10.5517 / \mathrm{ccsf0bj}$.

7. Purkayastha, P. Theoretical Modelling for the Ground State Rotamerisation and Excited State Intramolecular Proton Transfer of 2-(2'-hydroxyphenyl)oxazole, 2-(2'hydroxyphenyl)imidazole, 2-(2'-hydroxyphenyl)thiazole and Their Benzo Analogues / P. Purkayastha, N. Chattopadhyay // Int. J. Mol. Sci. - Vol. 4. - 2003. - P. 335 - 361.

8. Pijeau, S. Excited-State Dynamics of 2-(2'-Hydroxyphenyl)benzothiazole: Ultrafast Proton Transfer and Internal Conversion / S. Pijeau, D. Foster, E. G. Hohenstein // J. Phys. Chem. A. - Vol. 121. - 2017. - P. 4595 - 4605.

9. Syetov, Y. Lattice vibrations and disorder in crystalline benzoxazoles undergiong exited state intramolecular proton transfer: DFTB modeling / Y. Syetov // Journal of Physics and Electronics. - 2018. - Vol. 26(1). - P. $57-62$.

10. Aradi, B. DFTB+, a Sparse Matrix-Based Implementation of the DFTB Method / B. Aradi, B. Hourahine, Th. Frauenheim // J. Phys. Chem. A. - Vol. 111. 2007. - P. $5678-5684$.

11. Gaus, M. DFTB3: Extension of the Self-Consistent-Charge Density-Functional Tight-Binding Method (SCC-DFTB) / M. Gaus, Q. Cui, M. Elstner // J. of Chem. Theory Comput. - 2011. - Vol. 7. - P. 931 - 948.

12. Syetov, Y. Low-frequency Raman spectrum of crystalline 2-(2'hydroxyphenyl)benzoxazole and density-functional based tight-binding phonon calculations / Y. Syetov // Ukr. J. Phys. Opt. - 2017. - Vol. 18, No. 2. - P. 67 - 76.

13. Schmidt, M.W. General Atomic and Molecular Electronic Structure System / M.W. Schmidt, K.K. Baldridge, J.A. Boatz, S.T. Elbert, M.S. Gordon, J.H. Jensen, S. Koseki, N. Matsunaga, K.A. Nguyen, S.J. Su, T.L. Windus, M. Dupuis, J.A. Montgomery // J. Comput. Chem. - 1993. - Vol. 14. - P. 1347 - 1363.

14. Gordon, M.C. Advances in electronic structure theory: GAMESS a decade later / M.C. Gordon and M.W. Schmidt // Chapter 41 in Theory and Applications of Computational Chemistry, the first forty years. Ed. by Dykstra C.E., Frenking G., Kim K.S. and Scuseria G.E. - Amsterdam: Elsevier. - 2005. - P. 1167 - 1189. 